This is the final peer-reviewed accepted manuscript of:

Vaginal microbiome and metabolome highlight specific signatures of bacterial vaginosis

by Beatrice Vitali, Federica Cruciani, Gianfranco Picone, Carola Parolin, Gilbert Donders, Luca Laghi

Eur J Clin Microbiol Infect Dis. 2015 Dec; 34(12):2367-76. Epub 2015 Sep 18.

The final published version is available online at:

https://doi.org/10.1007/s10096-015-2490-y

Rights / License: CC BY

The terms and conditions for the reuse of this version of the manuscript are specified in the publishing policy. For all terms of use and more information see the publisher's website. 


\section{Vaginal microbiome and metabolome highlight specific signatures of bacterial vaginosis}

2

3

4 Beatrice Vitali · Federica Cruciani · Gianfranco Picone · Carola Parolin · Gilbert Donders · Luca Laghi

6 Beatrice Vitali $\cdot$ Federica Cruciani $\cdot$ Carola Parolin

7 Department of Pharmacy and Biotechnology, University of Bologna, Via San Donato 19/2, 40127

8 Bologna, Italy

9

10 Gianfranco Picone $\cdot$ Luca Laghi

11 Department of Agro-Food Science and Technology, University of Bologna, P.za Goidanich 60, 47522

12 Cesena, Italy

13

14 Gilbert Donders

15 Department of Obstetrics and Gynecology, General Hospital Heilig Hart, Kliniekstraat 45, 3300

16 Tienen, and University Hospital Antwerp, Wilrijkstraat 10, 2650 Edegem, Belgium

17

18 Corresponding author: Beatrice Vitali, Department of Pharmacy and Biotechnology, University of

19 Bologna, Via San Donato 19/2, 40127, Bologna, Italy.

20 Tel: +39 051 2088750; Fax: +39 0512099734

21 E-mail: $\underline{\text { b.vitali@unibo.it }}$

22

23 Federica Cruciani present address: CRA-CER S.S. 673 Km 25+200, 71122 Foggia, Italy. 


\section{Abstract}

Purpose. In the present study we sought to find novel bacterial and metabolic hallmarks for bacterial vaginosis $(\mathrm{BV})$.

Methods. We studied the vaginal microbiome and metabolome of vaginal fluids from BV-affected patients $(n=43)$ and healthy controls $(n=37)$ by means of an integrated approach based on quantitative PCR (qPCR) and proton Nuclear Magnetic Resonance $\left({ }^{1} \mathrm{H}-\mathrm{NMR}\right)$. The correlations between the clinical condition and vaginal bacterial communities were investigated by principal component analysis (PCA). To define the metabolomics signatures of BV, 100 discriminant analysis by projection on latent structure (PLS-DA) models were calculated.

Results. Bacterial signatures distinguishing the health condition and BV were identified by qPCR. $L$. crispatus strongly featured the healthy vagina while increased concentrations of Prevotella, Atopobium and Mycoplasma hominis specifically marked the infection. ${ }^{1} \mathrm{H}-\mathrm{NMR}$ analysis has led to the identification and quantification of 17 previously unreported molecules. BV was associated with changes in the concentration of metabolites belonging to the families of amines, organic acids, short chain fatty acids, amino acids, nitrogenous bases and monosaccharides. In particular, maltose, kynurenine and NAD+ primarily characterized the healthy status while nicotinate, malonate and acetate were the best metabolic hallmarks of BV.

Conclusions. This study helps to better understand the role of the vaginal microbiota and metabolome in the development of BV infection. We propose a molecular approach for diagnosis of BV based on quantitative detection in vaginal fluids of Atopobium, Prevotella and Mycoplasma hominis, and nicotinate, malonate and acetate by combining qPCR and ${ }^{1} \mathrm{H}-\mathrm{NMR}$.

Keywords: vaginal microbiome; vaginal metabolome; bacterial vaginosis; lactobacilli; qPCR; ${ }^{1} \mathrm{H}-\mathrm{NMR}$ 
The microbiota of the human vagina can significantly impact the health of women, their fetuses and newborn infants [1]. The vaginal microbiota of healthy reproductive age women is dominated by Lactobacillus species. Five distinct vaginal bacterial biotypes, characterized by the dominance of $L$. crispatus, L. gasseri, L. iners, L. jensenii, or an increased proportion of other strictly anaerobic bacteria, were described $[2,3]$. Lactobacilli play key protective roles through different mechanisms, such as production of various antibacterial compounds, co-aggregation, competitive exclusion and immunomodulation [4-6].

Bacterial vaginosis (BV) is a common polymicrobial disorder of the vaginal microbiota characterized by loss of lactobacilli and increasing numbers of anaerobes and gram-negative rods $[7,8]$. BV is associated with adverse outcomes, such as ascending reproductive tract infections, enhanced acquisition of HIV and other sexually transmitted diseases, spontaneous abortion and preterm birth [912]. Both a defined etiology and optimal treatment strategies for BV have remained elusive. BV is typically diagnosed using either the Nugent scoring method [13] that examines bacterial composition via a Gram smear or the Amsel criteria [14] that considers factors such as presence of discharge, amine production, presence of clue cells and a vaginal $\mathrm{pH}$ greater than 4.5 . The microbiology of BV has been better characterized through microbiome studies based on 16S rRNA gene-directed PCR assays [15]. Although these approaches are able to provide a comprehensive understanding of the bacterial community membership, they are not able to determine the changes occurring in the vaginal environment at a metabolic level. Metabolomics analyzes complex systems, using high-throughput analytical methods, such as NMR spectroscopy that allows robust and sensitive identification of metabolites produced by microbes and host cells. This tool allows researchers to

70 determine the effects caused by perturbations on the host's metabolic profile by analyzing the presence 
and quantity of thousands of metabolites simultaneously. Metabolites that are significantly affected by experimental variables can be identified by multivariate statistics and placed into the larger context of how the host was affected overall [16-19].

In the perspective to discern potential novel determinants of BV, we investigated the relationship between bacterial community composition and metabolic profiles of healthy and BV-associated vaginal ecosystems by means of quantitative PCR (qPCR) of bacterial 16S rRNA genes and proton Nuclear Magnetic Resonance $\left({ }^{1} \mathrm{H}-\mathrm{NMR}\right)$-based metabolomics.

\section{Materials and Methods}

Study participants and sample preparation

Subjects for this study were a cohort of 80 Belgian pre-menopausal, non-pregnant women, aged between 18 and 50 years (mean age: 37 ). The enrolled women belonged to two groups: BV-affected patients $(n=43)$ presenting positivity for at least three of four Amsel's criteria and a Nugent score $>3$, and age-matched healthy subjects $(n=37)$ who had no signs of vaginal tract infection and had never had BV. All women tested negative to Candida, sexually transmitted infections and abnormal findings on cervical Pap smears. They signed an informed consent in accordance with the approval and guidelines of the Ethics committee of the Heilig Hart Hospital of Tienen, Belgium.

Standardized vaginal fluids were collected by flushing and re-aspirating $2 \mathrm{ml}$ of saline through a 22gauge needle in the left, central, and right upper vaginal vaults [20] and stored at $-80^{\circ} \mathrm{C}$ until use.

Vaginal samples were centrifuged at $9,500 \times g$ for $15 \mathrm{~min}$. The supernatants were used for metabolomic analysis, while the pellets were processed for DNA isolation by using a DNeasy blood and tissue kit (Qiagen, Hilden, Germany) [21, 22]. DNA amount was quantified using NanoDrop ND- 
1000 (NanoDrop ${ }^{\circledR}$ Technologies, Wilmington, DE).

96

97 Quantitative PCR (qPCR)

98

99 qPCR was performed on DNA samples extracted from the vaginal fluids using a LightCycler

100 instrument (Roche, Mannheim, Germany) and SYBR Green I as the reporter fluorophore. Genus- or

101 species- specific primer sets targeted to 16S rRNA gene or 16S-23S rRNA spacer region were used to

102 amplify bacteria belonging to L. crispatus [23], L. iners [24], L. gasseri [25], L. jensenii [24],

103 Gardnerella vaginalis [26], Atopobium [27], Prevotella [28], Veillonella [29], Mycoplasma hominis

104 [23] and Mobiluncus [30].

105 Amplifications were carried out in a final volume of $20 \mu \mathrm{l}$ containing each primer at $0.5 \mu \mathrm{M}, 4 \mu \mathrm{l}$ of

106 LightCycler-FastStart DNA Master SYBR green I (Roche) and $2 \mu 1$ of template. The thermal cycling

107 conditions were optimized in the present study (Table S1, supplemental material). DNAs extracted

108 from L. crispatus DSM 20584, L. gasseri DSM 20243, L. jensenii DSM 20557, L. iners DSM 13335,

109 Gardnerella vaginalis ATCC 14018, Prevotella bivia ATCC 29303, Veillonella parvula ATCC 10790,

110 Atopobium vaginae ATCC BAA-55, Mycoplasma hominis DSM 19104, and Mobiluncus curtisii ATCC

11143063 were used as standards for PCR quantification. The efficiency of qPCR was between $90 \%$ and

$112100 \%$, and correlation coefficients for genomic DNA standards were $>0.99$. The sensitivity of qPCR

113 assays ranged from $10^{-4}$ to $10^{-1} \mathrm{ng}$ of target DNA. Melting curve analysis was carried out to confirm

114 that the PCR products from vaginal samples and standards had identical melting points.

115 DNA samples were amplified in triplicate for each primer set. Data were expressed as log ng of DNA

116 of the target genus or species per $\mu \mathrm{g}$ of total DNA extracted from the vaginal sample.

117

$118 \quad{ }^{1} \mathrm{H}-\mathrm{NMR}$ analysis 
120 One ml of vaginal supernatant was added to $160 \mu \mathrm{l}$ of a $\mathrm{D}_{2} \mathrm{O}$ solution of 3-(trimethylsilyl)-propionic-

$1212,2,3,3-\mathrm{d} 4$ acid sodium salt (TSP) $6.25 \mathrm{mM}$ and the $\mathrm{pH}$ was adjusted to 7.00 with the addition of $\mathrm{HCl}$ or

$122 \mathrm{NaOH}(0.5 \mathrm{M}) .{ }^{1} \mathrm{H}-\mathrm{NMR}$ spectra were recorded at $298 \mathrm{~K}$ with an AVANCE spectrometer (Bruker,

123 Milan, Italy) operating at a frequency of $600.13 \mathrm{MHz}$, equipped with an autosampler with 60 holders

124 [17]. Each spectrum was acquired using $32 \mathrm{~K}$ data points over a 7,211.54 Hz spectral width and adding

125256 transients. A recycle delay of $5 \mathrm{~s}$ and a $90^{\circ}$ pulse of $11.4 \mathrm{~s}$ were set up. Acquisition time (2.27 s)

126 and recycle delay were adjusted to be 5 times longer than the longitudinal relaxation time of the protons

127 under investigation, which was considered to be not longer than $1.4 \mathrm{~s}$.

$128{ }^{1} \mathrm{H}-\mathrm{NMR}$ spectra baseline was adjusted by means of the simultaneous peak detection [31] and baseline

129 correction algorithm (SPDBC) implemented in the baseline R package [32]. Signals misalignments

130 were compensated by i-Coshift algorithm [33]. Differences in water concentration were compensated

131 by probabilistic quotient normalization procedure (PQN) [34]. The signals with an intensity of at least

132 five times that of noise were assigned by comparing their chemical shift and multiplicity with the

133 literature [17] and Chenomx software data bank (Chenomx Inc., Canada, ver 8.1). Their area was 134 employed for molecular quantification.

136 Statistical analysis

138 Statistical analyses were performed using R computational language [35]. Similarities among the

139 analyzed samples, as well as trends in the bacterial communities or metabolome profiles, were

140 investigated by means of principal components analysis (PCA) applied to the mean centered data.

141 Differences in bacterial amounts, metabolites concentrations and PCA data were analyzed using

142 Wilcoxon's signed rank test and Anova based on Tukey contrast [36]. A $P$ value $<0.05$ was considered 
statistically significant. In addition, to better define the metabolomics signatures of BV, 100

144 discriminant analysis by projection on latent structure (PLS-DA) models in their sparse version were

145 calculated by means of mixOmics R package [37]. PLS-DA algorithm builds linear models with each

146 molecule concentration in order to maximize the possibility to predict BV presence. In its sparse

147 version, the most informative molecules are progressively added to the model until the best

148 compromise between prediction ability and model simplicity is reached. The robustness of the models

149 was expressed in terms of correct classification rate (CCR), that is the ratio between sum of true

150 positives and negatives predictions and the total number of samples.

\section{Results}

153

154 Distribution of bacterial communities

155

156 A qualitative analysis of the microbiota composition in healthy and BV-affected women is represented

157 in Fig. 1. The frequency of occurrence of Lactobacillus species and BV-related bacteria represented the

158 first element to clearly distinguish the two vaginal ecosystems (Fig. 1a). Healthy women primarily

159 harboured lactobacilli. Among these, L. crispatus was the most frequently detected bacterium

160 (70.27\%), followed by L. gasseri (45.94\%), L. iners (24.32\%) and L. jensenii (18.92\%). The detection

161 rate of $L$. iners was higher in BV-affected women (62.79\%) than in healthy controls. The most

162 frequently BV-related bacteria detected in healthy women were Prevotella (67.57\%), M. hominis

163 (62.16\%) and Atopobium (54.05\%). G. vaginalis was found in a small percentage (8.11\%), while

164 Veillonella and Mobiluncus were not detected. BV patients possessed a diverse array of bacteria, with

165 the most frequent genera and species being Atopobium (100\%), G. vaginalis (97.67\%), Prevotella

166 (97.67\%) and M. hominis (83.72\%). Despite Veillonella (41.86\%) and Mobiluncus (23.26\%) have been 
found at a lower frequency than the other BV-related bacteria, their presence seemed to indicate unequivocally the existence of BV disorder. Differences in frequency of occurrence between healthy and BV-affected women were significant for all bacterial groups $(P<0.05)$, except for $L$. jensenii. The relative abundance of the main Lactobacillus species associated with human vaginal mucosa has been explored as an additional criterion for distinguishing between health and BV (Fig. 1b). The majority of women was characterized by the presence of a single Lactobacillus species. For women who harboured more than one Lactobacillus species, the predominant species was identified based on the bacterial amount calculated by qPCR. The vaginal microbiota of the totality of healthy women was colonized by at least one Lactobacillus species. L. crispatus (59\%) highly prevailed over other lactobacilli, followed by L. iners (22\%), L. gasseri (16\%), and L. jensenii (3\%). Unlike health condition, the vaginal microbiota of a fair number of BV patients was not colonized by any of the four main Lactobacillus species (N: 33\%) and L. iners was the predominant species (63\%). The microbiota of a low percentage of BV-affected women was dominated by L. gasseri (5\%), while L. jensenii and $L$. crispatus were never predominant.

181

Quantification of Lactobacillus species and BV-related bacteria

183

184 The qPCR results are summarized in box blots representing concentrations of specific DNA of Lactobacillus species and BV-related bacteria in vaginal samples of healthy controls and BV patients (Fig. 2).

187 Regarding lactobacilli, only L. crispatus showed a significant difference between healthy and BV 188 women: the amount of this species was higher in healthy group (7.75 log ng/ $\mu \mathrm{g})$ than in BV group 189 (6.47 $\log \mathrm{ng} / \mu \mathrm{g})(P<0.0001)$. As already reported [38], L. iners had the highest median concentration

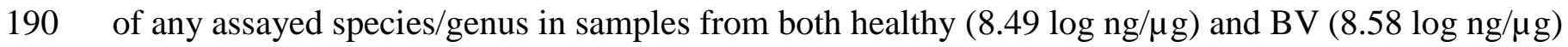


women.

192 With respect to BV-related bacteria, the median concentrations of Atopobium (H: $0.13 \log \mathrm{ng} / \mu \mathrm{g}$; BV:

$1931.96 \log \mathrm{ng} / \mu \mathrm{g} ; P<0.0001)$, Prevotella $(\mathrm{H}:-1.24 \log \mathrm{ng} / \mu \mathrm{g} ; \mathrm{BV}: 1.20 \log \mathrm{ng} / \mu \mathrm{g} ; P<0.0004)$ and $M$.

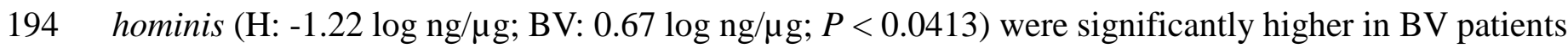

195 compared to healthy controls. The amount of $G$. vaginalis did not vary significantly in the two groups

196 of women. Veillonella (-1.07 log $\mathrm{ng} / \mu \mathrm{g})$ and Mobiluncus (1 log $\mathrm{ng} / \mu \mathrm{g})$ were identified only in BV cases

197 at low concentrations.

198

199

Metabolic profiles

200

201 We sought a metabolic description of BV compared to health condition. 55 signals ascribable to single 202 molecules were identified (Table S2, supplemental material). 44 of these molecules were identified and 203 quantified, while other 6 were identified, but not reliably quantified, due to signals superimpositions or 204 artifacts introduced by signal alignment procedure. Concerning citrate, BV condition was not 205 associated to a variation in concentration, but to a shift in signals in the spectrum, a well-known 206 behavior caused by oscillations in divalent cations concentrations [39]. We identified and quantified 17 207 previously unreported small molecules, including the organic acids malonate and isovalerate, the 208 amino acids histidine, taurine and aspartate, the nitrogenous bases NAD+ and inosine.

209 Among the identified and quantified molecules, 32 significantly varied in concentration between 210 healthy and BV women $(P<0.05)$. These metabolites belong to the families of amines, organic acids, 211 short chain fatty acids (SCFAs), amino acids, nitrogenous bases and monosaccharides (Table 1). BV 212 was associated with a general increase of amines, organic acids and various SCFAs. BV was also 213 characterized by a general decrease of protein amino acids, with the exception of proline. Within the 214 other categories of metabolites no uniform trend of variation was observed. 
216 Microbiome-metabolome correlation

218 The correlations between the clinical condition and vaginal bacterial communities have been studied by 219 means of a PCA built on the qPCR data related to Lactobacillus species and BV-related bacteria (Fig. 220 S1, supplemental material). The first two PCs accounted for the $32.3 \%$ of the whole variance of the 221 investigated samples.

222 Changes in bacterial populations were highlighted by plotting the medians of the two groups of women 223 (H and BV). $\mathrm{H}$ and BV groups were significantly different $(P<0.05)$, showing that most of the 224 variability was due to the onset of BV condition. BV samples were more widely distributed in the 225 multidimensional space compared to healthy samples, indicating a greater inter-individual variability of microbiome and metabolome, as previously hypothesized [17, 40]. The vaginal microbiota of control women was dominated by L. crispatus, L. gasseri and L. jensenii with the former most strongly characterizing the state of health. L. iners was shifted toward the disease condition, suggesting its correlation with the development of BV. The bacterial groups that primarily featured women with BV were Prevotella and Atopobium, followed by M. hominis. Conversely, Mobiluncus and Veillonella, which were identified in small quantities only in presence of $\mathrm{BV}$, played a minor role as biomarkers of the disease. The results of PCA show that concentration is a relevant factor in determining a distinctive feature of BV in terms of diagnostic applications. G. vaginalis did not appear as one of the species most closely associated with BV.

The implications of BV on vaginal metabolome were investigated by building 100 sPLS-DA models on the metabolites that significantly varied in BV women compared to healthy controls (Fig. 3). At least in 90 times the variable reduction algorithm selected maltose, kynurenine, nicotinate, malonate, acetate and $\mathrm{NAD}^{+}$as the most important metabolites in discriminating vaginal health from $\mathrm{BV}$. In particular, 
maltose, kynurenine and NAD+ were distinctive of healthy condition while nicotinate, malonate, and 240 acetate appeared to be specific hallmarks of BV. These molecules were also characterized by median 241 variable influence on projections (VIP) coefficients higher than 1, feature that was shared with sarcosine and phenylalanine. PLS-DA model built on these 6 molecules only gave a percentage of 0.11 $243 \pm 0.42$ (mean \pm standard deviation) of misclassified samples pertaining to BV-affected women, and no 244 misclassified test samples pertaining to healthy women. This optimal performance was obtained in all 245 cases, including women dominated by L. iners. This means that, despite these women presented a BV246 like microbiome, the conditions regulating the concentration of maltose, kynurenine, nicotinate, 247 malonate, acetate and $\mathrm{NAD}^{+}$represent a common trait of $\mathrm{BV}$ onset and could be reliably used as 248 biomarkers and to better understand the biochemical bases of this disease.

250 Correlation between Lactobacillus species, BV-related bacteria and metabolites

252 In order to go into deeper detail about the health-promoting potential of L. crispatus, L. iners, L. 253 gasseri and L. jensenii, the correlations between the dominance of each Lactobacillus species and the 254 vaginal microbiome and metabolome were analyzed by PCA (Fig. S2, supplemental material). In the 255 biplot describing the correlations between the dominant Lactobacillus species and BV-related bacteria, 256 PC1 and PC2 accounted for the $47.4 \%$ of the whole variance of the investigated samples (Fig. S2a). 257 Medians of the four healthy groups were superimposed while the median of BVn was significantly 258 different from the medians of $\mathrm{BVi}$ and $\mathrm{BVg}(P<0.05)$, showing that the variability of BV samples was 259 not only associated to the development of BV but also to the presence of lactobacilli. The more 260 different microbiota from health condition was found in BVn, followed by BVi. An intermediate 261 position in the two-dimensional space was occupied by BVg.

262 The six molecules best discriminating healthy condition from BV allowed to obtain a visual 
correlations between the predominance of each Lactobacillus species and metabolome, as depicted in

264 Fig. S2b. PC1 and PC2 accounted for the $67.9 \%$ of the whole variance of the investigated samples. The 265 distribution in the PC plane of the samples from BV-affected women closely reproduced the trends 266 observed in Fig. S2a, with BVg samples appearing as most similar to the healthy samples, BVn 267 samples most different, and L. iners prevalence giving rise on average to an intermediate condition.

\section{Discussion}

270

271 In the present study we sought to identify correlations between the vaginal colonization of certain 272 bacterial populations and metabolic profiles, in order to find novel hallmarks of BV.

273 Our results support previous studies that BV is associated with dramatic compositional changes in the 274 vaginal microbiota, i.e. depletion of lactobacilli in conjunction with colonization of many diverse 275 bacteria, mainly strict anaerobes [7, 20, 39, 41, 42]. Healthy women primarily harbored lactobacilli: $L$. 276 crispatus was the most frequent species, detected at significantly higher concentration with respect to 277 BV patients. The frequency of occurrence of L. iners was higher in BV-affected women, confirming the 278 hypothesis that this species is common and abundant in vaginal communities characterized by high 279 concentrations of non-lactobacilli $[22,42]$. Other studies of perturbed vaginal microflora suggest that $L$. 280 iners might be a transitional species, colonizing after disturbances to the vaginal environment [43]. Compared to healthy controls, BV patients had in their vaginal microbiota higher prevalence of all the considered BV-related bacteria and significantly higher abundances of Atopobium, Prevotella and $M$. hominis. Because Atopobium, Prevotella and M. hominis were also frequently represented in healthy vagina (> 50\%), high levels rather than the mere presence of these bacteria can be considered hallmarks of BV disorder. G. vaginalis, which has long been considered distinctive of BV [44], in our study did not seem to be particularly useful for diagnostic purposes. In fact, neither the presence nor an increased 
concentration of this species were uniquely associated with the disease. The results of multivariate statistical analysis reiterated the role of $L$. crispatus in strongly featuring the healthy vagina and the functions of Prevotella, Atopobium and M. hominis as specific microbio-markers of BV disorder. Conversely, Mobiluncus and Veillonella, even though highly specific for BV, seemed to play a minor role in marking the disease, probably because the majority of $\mathrm{BV}$-affected women did not harbor these bacteria. It remains to be elucidated whether Mobiluncus/Veillonella positive BV expresses a subtype of BV with specific features and pathogenicity. Notably, PCA highlighted the relevance of bacterial concentration in determining a distinctive feature for proper diagnosis of BV.

The molecular data descriptive of the vaginal microbiome have been integrated with the ${ }^{1} \mathrm{H}-\mathrm{NMR}$ data descriptive of the metabolic activities of the host-bacteria meta-organisms, in order to obtain a comprehensive picture of the evolution of the vaginal ecosystem associated with the infection. This led to the identification and quantification of 17 previously unreported small molecules, including the organic acids malonate and isovalerate, the amino acids histidine, taurine and aspartate, the nitrogenous bases $\mathrm{NAD}^{+}$and inosine.

BV was associated with changes in several metabolites belonging to the families of amines, organic acids, short chain fatty acids, amino acids, nitrogenous bases and monosaccharides. Most of these molecules are known to be modulated by the balance between lactobacilli and BV-related bacteria, such as biogenic amines, SCFAs and organic acids [17, 45-49]. In particular, we have observed in BV cases a general increase of amines, including tyramine, trimethylamine and cadaverine, which are considered the main responsible for the fishy odor of the vaginal discharge $[8,19]$. Various SCFAs were significantly higher in BV-positive women, which is not surprising given that BV is characterized by the outgrowth of mostly anaerobic bacteria. It has been suggested that SCFAs may be involved in recruitment and activation of the innate immune cells in the female genital tract [47]. This is in agreement with the recent finding that $\mathrm{BV}$ is associated with an increase of proteins involved in the 
311 innate immune response [50]. Organic acids were detected at higher concentrations in the vaginal fluids 312 of BV-positive women, as previously noted especially for acetate and succinate, typical metabolites 313 produced by Prevotella and Mobiluncus spp. [45]. In the present work, malonate also assumes a 314 particular emphasis being together acetate the organic acids that mostly differ BV from healthy state. 315 BV was also characterized by a decrease of certain protein amino acids, probably due to their 316 decarboxylation to biogenic amines. The high availability of maltose in healthy vagina can be regarded 317 as the principal cause of the overgrowth of lactobacilli. It could be attributed to an efficient synthesis of 318 glycogen and/or secretion of $\alpha$-amylase by healthy vaginal epithelium. In fact, the human $\alpha$-amylase is 319 primarily responsible for the digestion of vaginal glycogen, making available glycogen-breakdown 320 products, including maltose [51]. On the other hand, we found an increase of glucose concentration in $321 \mathrm{BV}$-affected patients. This finding is in agreement with previous studies reporting the association of 322 dietary indices, i.e. glycemic load, with BV acquisition and persistence [52]. The high level of glucose 323 could favor the glycolysis which involves the consumption of $\mathrm{NAD}^{+}$.

324 In conclusion, our work may help to better understand the role of the vaginal microbiota and 325 metabolome in BV infection. The identification of bacterial and metabolic markers described here is a 326 prerequisite for the design of new diagnostic kits, which, being based on molecular parameters, are 327 likely to be endowed with greater sensitivity and reproducibility compared to the methods of diagnosis 328 currently used in clinical practice (Amsel and Nugent). Specifically, we propose a molecular tool for 329 diagnosis of BV based on quantitative detection in vaginal fluids of Atopobium, Prevotella and 330 Mycoplasma hominis, and malonate, acetate, and nicotinate by combining $\mathrm{qPCR}$ and ${ }^{1} \mathrm{H}-\mathrm{NMR}$. The 331 combination of these selected bacterial/ metabolic hallmarks could provide the best diagnostic test in 332 terms of specificity, allowing to correctly diagnose cases of infection reducing false positive rate. 333 Regarding sensitivity, such a combination maintains the peculiarities of qPCR and ${ }^{1} \mathrm{H}-\mathrm{NMR}$ 334 techniques, that are able to detect bacterial DNA and metabolites at concentrations below the ng and 
$\mathrm{mmol} / \mathrm{l}$, respectively.

336

337 Acknowledgments This study was supported by MIUR (Ministry of Instruction, University and 338 Research, Italy).

339

340 Conflicts of interest The authors declare that they have no conflict of interest.

342 Ethical approval All procedures performed in studies involving human participants were in

343 accordance with the ethical standards of the institutional and/or national research committee and with 344 the 1964 Helsinki declaration and its later amendments or comparable ethical standards. 
1. Hillier SL, Krohn MA, Cassen E, Easterling TR, Rabe LK, Eschenbach DA (1995) The role of bacterial vaginosis and vaginal bacteria in amniotic fluid infection in women in preterm labor with intact fetal membranes. Clin Infect Dis. 20 Suppl 2:S276-278.

2. Drell T, Lillsaar T, Tummeleht L, Simm J, Aaspõllu A, Väin E, Saarma I, Salumets A, Donders GG, Metsis M (2013) Characterization of the vaginal micro- and mycobiome in asymptomatic reproductive-age Estonian women. PLoS One 8(1):e54379.

3. Ravel J, Gajer P, Abdo Z, Schneider GM, Koenig SS, McCulle SL, Karlebach S, Gorle R, microbiome of reproductive-age women. Proc Natl Acad Sci USA 108 Suppl 1:4680-4687.

4. Kaewsrichan J, Peeyananjarassri K, Kongprasertkit J (2006) Selection and identification of anaerobic lactobacilli producing inhibitory compounds against vaginal pathogens. FEMS Immunol Med Microbiol 48:75-83.

5. Klebanoff SJ, Hillier SL, Eschenbach DA, Waltersdorph AM (1991) Control of the microbial flora of the vagina by $\mathrm{H}_{2} \mathrm{O}_{2}$-generating lactobacilli. J Infect Dis 164:94-100.

6. Reid G, Younes JA, Van der Mei HC, Gloor GB, Knight R, Busscher HJ (2011) Microbiota restoration: natural and supplemented recovery of human microbial communities. Nat Rev Microbiol 9:27-38.

7. Fredricks DN, Fiedler TL, Marrazzo JM (2005) Molecular identification of bacteria associated with bacterial vaginosis. N Engl J Med 353:1899-1911.

8. Sobel JD (2000) Bacterial vaginosis. Annu Rev Med 51:349-356.

9. Atashili J, Poole C, Ndumbe PM, Adimora AA, Smith JS (2008) Bacterial vaginosis and HIV acquisition: a meta-analysis of published studies. AIDS 22:1493-1501.

10. Brotman RM, Klebanoff MA, Nansel TR, Yu KF, Andrews WW, Zhang J, Schwebke JR (2010) 
Bacterial vaginosis assessed by gram stain and diminished colonization resistance to incident gonococcal, chlamydial, and trichomonal genital infection. J Infect Dis 202:1907-1915.

11. Donders GG, Van Bulck B, Caudron J, Londers L, Vereecken A, Spitz B (2000) Relationship of bacterial vaginosis and mycoplasmas to the risk of spontaneous abortion. Am J Obstet Gynecol 183:431-437.

12. Hillier SL, Martius J, Krohn M, Kiviat N, Holmes KK, Eschenbach DA (1988) A case-control 319:972-978.

13. Nugent RP, Krohn MA, Hillier SL (1991) Reliability of diagnosing bacterial vaginosis is improved by a standardized method of gram stain interpretation. J Clin Microbiol 29:297-301.

14. Amsel R, Totten PA, Spiegel CA, Chen KC, Eschenbach D, Holmes KK (1983) Nonspecific vaginitis. Diagnostic criteria and microbial and epidemiologic associations. Am J Med 74:1422.

15. Lamont RF, Sobel JD, Akins RA, Hassan SS, Chaiworapongsa T, Kusanovic JP, Romero R (2011) The vaginal microbiome: new information about genital tract flora using molecular based techniques. BJOG 118:533-549.

16. Coen M, O'Sullivan M, Bubb WA, Kuchel PW, Sorrell T (2005) Proton nuclear magnetic resonance-based metabonomics for rapid diagnosis of meningitis and ventriculitis. Clin Infect Dis $41: 1582-1590$.

17. Laghi L, Picone G, Cruciani F, Brigidi P, Calanni F, Donders G, Capozzi F, Vitali B (2014) Rifaximin modulates the vaginal microbiome and metabolome in women affected by bacterial vaginosis. Antimicrob Agents Chemother 58:3411-3420.

18. Urbanczyk-Wochniak E, Luedemann A, Kopka J, Selbig J, Roessner-Tunali U, Willmitzer L, Fernie AR (2003) Parallel analysis of transcript and metabolic profiles: a new approach in 
systems biology. EMBO Rep 4:989-993.

19. Yeoman CJ, Thomas SM, Miller ME, Ulanov AV, Torralba M, Lucas S, Gillis M, Cregger M, Gomez A, Ho M, Leigh SR, Stumpf R, Creedon DJ, Smith MA, Weisbaum JS, Nelson KE, Wilson BA, White BA (2013) A multi-omic systems-based approach reveals metabolic markers of bacterial vaginosis and insight into the disease. PLoS One 8(2):e56111.

20. Vitali B, Pugliese C, Biagi E, Candela M, Turroni S, Bellen G, Donders GG, Brigidi P (2007) Dynamics of vaginal bacterial communities in women developing bacterial vaginosis, candidiasis, or no infection, analyzed by PCR-denaturing gradient gel electrophoresis and realtime PCR. Appl Environ Microbiol 73:5731-5741.

21. Vitali B, Biagi E, Brigidi P (2012) Protocol for the use of PCR-denaturing gradient gel 404 electrophoresis and quantitative PCR to determine vaginal microflora constitution and pathogens in bacterial vaginosis. In: MacKenzie CR, Henrich B (eds.) Diagnosis of Sexually Transmitted Diseases. Springer, New York, vol 903, pp 177-193.

22. Cruciani F, Brigidi P, Calanni F, Lauro V, Tacchi R, Donders G, Peters K, Guaschino S, Vitali B (2012) Efficacy of rifaximin vaginal tablets in treatment of bacterial vaginosis: a molecular characterization of the vaginal microbiota. Antimicrob Agents Chemother 56:4062-4070.

23. Zozaya-Hinchliffe M, Lillis R, Martin DH, Ferris MJ (2010) Quantitative PCR assessments of bacterial species in women with and without bacterial vaginosis. J Clin Microbiol 48:18121819.

24. De Backer E, Verhelst R, Verstraelen H, Alqumber MA, Burton JP, Tagg JR, Temmerman M, Vaneechoutte M (2007) Quantitative determination by real-time PCR of four vaginal Lactobacillus species, Gardnerella vaginalis and Atopobium vaginae indicates an inverse relationship between L. gasseri and L. iners. BMC Microbiol 7:115. 
analysis of diverse Lactobacillus species present in advanced dental caries. J Clin Microbiol 42: 3128-3136.

26. Zariffard MR, Saifuddin M, Sha BE, Spear GT (2002) Detection of bacterial vaginosis-related organisms by real-time PCR for Lactobacilli, Gardnerella vaginalis and Mycoplasma hominis. FEMS Immunol Med Microbiol 34: 277-281.

27. Matsuki T, Watanabe K, Fujimoto J, Takada T, Tanaka R (2004) Use of 16S rRNA gene-targeted group-specific primers for Real-Time PCR analysis of predominant bacteria in human feces. Appl Environ Microbiol 70:7220-7228.

28. Matsuki T, Watanabe K, Fujimoto J, Miyamoto Y, Takada T, Matsumoto K, Oyaizu H, Tanaka R (2002) Development of 16S rRNA-gene-targeted group-specific primers for the detection and identification of predominant bacteria in human feces Appl Environ Microbiol 68:5445-5451.

29. Rinttilä T, Kassinen A, Malinen E, Krogius L, Palva A (2004) Development of an extensive set of 16S rDNA-targeted primers for quantification of pathogenic and indigenous bacteria in faecal samples by real-time PCR. J Appl Microbiol 97:1166-1177.

30. Tiveljung A, Forsum U, Monstein HJ (1996) Classification of the genus Mobiluncus based on comparative partial 16S rRNA gene analysis. Int J Syst Bacteriol 46:332-336.

31. Coombes KR, Fritsche HA, Clarke C, Chen J-N, Baggerly KA, Morris JS, Xiao LC, Hung MC, Kuerer HM (2003) Quality control and peak finding for proteomics data collected from nipple spirate fluid by surface-enhanced laser desorption and ionization. Clin Chem 49:1615-1623.

32. Liland KH, Almøy T, Mevik BH (2010) Optimal choice of baseline correction for multivariate calibration of spectra. Appl Spectrosc 64:1007-1016.

33. Savorani F, Tomasi G, Engelsen SB (2010) icoshift: A versatile tool for the rapid alignment of 1D NMR spectra. J Magn Reson 202:190-202.

34. Dieterle F, Ross A, Schlotterbeck G, Senn H. (2006) Probabilistic quotient normalization as 
robust method to account for dilution of complex biological mixtures. Application in ${ }^{1} \mathrm{H}$ NMR metabonomics. Anal Chem 78:4281-4290.

35. Ihaka R, Gentleman R (1996) R: a language for data analysis and graphics. J Comput Graph Stat 5:299-314.

36. Hothorn T, Bretz F, Westfall P (2008) Simultaneous inference in general parametric models. Biom J 50:346-363.

37. Yao F1, Coquery J, Lê Cao KA (2012) Independent Principal Component Analysis for biologically meaningful dimension reduction of large biological data sets. BMC Bioinformatics $13: 24$

38. Shipitsyna E, Roos A, Datcu R, Hallén A, Fredlund H, Jensen JS, Engstrand L, Unemo M, (2013) Composition of the vaginal microbiota in women of reproductive age-sensitive and specific molecular diagnosis of bacterial vaginosis is possible? PLoS One 8(4):e60670.

39. Spraul M, Schütz B, Humpfer E, Mörtter M, Schäfer H, Koswig S, Rinke P (2009) Mixture analysis by NMR as applied to fruit juice quality control. Magn Reson Chem 47 Suppl 1:S130S137.

40. Biagi E, Vitali B, Pugliese C, Candela M, Donders GG, Brigidi P (2009) Quantitative variations in the vaginal bacterial population associated with asymptomatic infections: a real-time polymerase chain reaction study. Eur J Clin Microbiol Infect Dis 28:281-285.

41. Turovskiy Y, Sutyak Noll K, Chikindas ML (2011) The aetiology of bacterial vaginosis. J Appl Microbiol 110:1105-1128.

42. Ling Z, Kong J, Liu F, Zhu H, Chen X, Wang Y, Li L, Nelson KE, Xia Y, Xiang C (2010) Molecular analysis of the diversity of vaginal microbiota associated with bacterial vaginosis. BMC Genomics 11:488.

43. Macklaim JM, Gloor GB, Anukam KC, Cribby S, Reid G (2011) At the crossroads of vaginal 
health and disease, the genome sequence of Lactobacillus iners AB-1. Proc Natl Acad Sci U S A 108 Suppl 1:4688-95.

44. Gardner HL, Dukes CD (1955) Haemophilus vaginalis vaginitis: a newly defined specific infection previously classified non-specific vaginitis. Am J Obstet Gynecol 69:962-976.

45. Al-Mushrif S, Eley A, Jones BM (2000) Inhibition of chemotaxis by organic acids from anaerobes may prevent a purulent response in bacterial vaginosis. J Med Microbiol 49:10231030.

46. Chaudry AN, Travers PJ, Yuenger J, Colletta L, Evans P, Zenilman JM, Tummon A (2004) Analysis of vaginal acetic acid in patients undergoing treatment for bacterial vaginosis. J Clin Microbiol 42:5170-5175.

47. Mirmonsef P, Gilbert D, Zariffard MR, Hamaker BR, Kaur A, Landay AL, Spear GT (2011) The effects of commensal bacteria on innate immune responses in the female genital tract. Am J Reprod Immunol 65:190-195.

48. Sobel JD, Karpas Z, Lorber A (2012) Diagnosing vaginal infections through measurement of biogenic amines by ion mobility spectrometry. Eur J Obstet Gynecol Reprod Biol 163:81-84.

49. Wolrath H, Forsum U, Larsson PG, Borén H (2001) Analysis of bacterial vaginosis-related amines in vaginal fluid by gas chromatography and mass spectrometry. J Clin Microbiol 39:4026-4031.

50. Cruciani F, Wasinger V, Turroni S, Calanni F, Donders G, Brigidi P, Vitali B (2013) Proteome profiles of vaginal fluids from women affected by bacterial vaginosis and healthy controls: outcomes of rifaximin treatment. J Antimicrob Chemother 68:2648-2659.

51. Spear GT, French AL, Gilbert D, Zariffard MR, Mirmonsef P, Sullivan TH, Spear WW, Landay A, Micci S, Lee BH, Hamaker BR (2014) Human $\alpha$-amylase present in lower-genital-tract mucosal fluid processes glycogen to support vaginal colonization by Lactobacillus. J Infect Dis 
210:1019-1028.

491 52. Thoma ME, Klebanoff MA, Rovner AJ, Nansel TR, Neggers Y, Andrews WW, Schwebke JR

492 (2011) Bacterial vaginosis is associated with variation in dietary indices. J Nutr 141:1698-1704.

493 


\section{Figure captions}

495

496 Fig. 1 Molecular analysis of the vaginal microbiota composition in healthy $(\mathrm{H})$ and BV-affected (BV)

497 women. (a) Frequency of occurrence of L. crispatus, L. iners, L. gasseri, L. jensenii, Atopobium, G. 498 vaginalis, Prevotella, Veillonella, Mobiluncus and M. hominis, calculated as the percentage of women 499 hosting each bacterial group. **, $P<0.01$; *, $P<0.05$. (b) Relative abundance of L. crispatus, L. iners, 500 L. gasseri and L. jensenii, calculated as percentage of women who hosted each species as dominant. 501 (the predominant species was identified based on the bacterial amount calculated by qPCR). N indicate 502 the percentage of women that is not colonized by any of the four analysed species 503

Fig. 2 Quantification of L. crispatus, L. iners, L. gasseri, L. jensenii, Atopobium, G. vaginalis, 505 Prevotella, Veillonella, Mobiluncus and M. hominis in healthy (H) and BV-affected (BV) women. qPCR data are expressed as log ng of DNA of the target genus or species per $\mu \mathrm{g}$ of total DNA extracted from the vaginal sample. The box for each bacterial group represents the interquartile range $\left(25^{\text {th }}\right.$ to $75^{\text {th }}$ percentile), and the line within this box is the median value. Bottom and top bars indicate the $10^{\text {th }}$ and $90^{\text {th }}$ percentiles, respectively. Outlier values are indicated (black circles). Only positive samples for each bacterial group analysed were considered. **, $P<0.01 ; *, P<0.05$

513 Fig. 3 Frequency of inclusion of the 32 molecules of Table 1 calculated by the sPLS-DA predictive 514 model. The median variable influence on projections (VIP) coefficients are indicated in parentheses 


\section{Supplemental material}

520 Fig. S1 Biplot of a PCA performed on the autoscaled qPCR data. Median values of the samples groups 521 corresponding to healthy and BV-affected women are indicated as H and BV. Empty circles and filled 522 squares indicate samples from healthy and BV women, respectively. Expl. Var, explained variance 523

524 Fig. S2 Biplot of a PCA performed on the autoscaled qPCR data related to BV-associated bacteria (a) 525 and metabolites selected by sPLS-DA model. (b) Median values of the samples groups corresponding 526 to healthy women dominated by L. crispatus, L. iners, L. gasseri, L. jensenii and BV-affected women 527 dominated by L. iners, L. gasseri or none of the four considered species are indicated as $\mathrm{Hc}, \mathrm{Hi}, \mathrm{Hg}, \mathrm{Hj}$, $528 \mathrm{BVi}, \mathrm{BVg}$ and BVn, respectively. Empty circles and filled squares indicate samples from healthy and 529 BV women, respectively. Expl. Var, explained variance 
530 Table 1 Concentration of metabolites which significantly varied $(P<0.05)$ in BV-affected patients

531 (BV) compared to healthy control women $(\mathrm{H})$

\begin{tabular}{|c|c|c|c|c|}
\hline Molecule & $\begin{array}{c}\text { Chemical shift } \\
\text { (ppm) }\end{array}$ & $\mathbf{H}$ & BV & $\begin{array}{c}\text { Variations in } \\
\text { BV }\end{array}$ \\
\hline \multicolumn{5}{|l|}{ Amines } \\
\hline Tyramine & 7.228 & $1.52 \times 10^{-02} \pm 1.29 \times 10^{-02}$ & $9.21 \times 10^{-02} \pm 1.02 \times 10^{-01}$ & $\uparrow$ \\
\hline Ethanolamine & 3.151 & $6.04 \times 10^{-02} \pm 1.85 \times 10^{-02}$ & $1.18 \times 10^{-01} \pm 5.29 \times 10^{-02}$ & $\uparrow$ \\
\hline TMA & 2.894 & $6.50 \times 10^{-04} \pm 7.13 \times 10^{-04}$ & $2.13 \times 10^{-02} \pm 1.99 \times 10^{-02}$ & $\uparrow$ \\
\hline Methylamine & 2.61 & $9.08 \times 10^{-04} \pm 8.91 \times 10^{-04}$ & $1.97 \times 10^{-02} \pm 2.12 \times 10^{-02}$ & $\uparrow$ \\
\hline Cadaverine & 1.722 & $1.64 \times 10^{-01} \pm 6.66 \times 10^{-02}$ & $3.80 \times 10^{-01} \pm 3.15 \times 10^{-01}$ & $\uparrow$ \\
\hline \multicolumn{5}{|l|}{ Organic acids } \\
\hline Formate & 8.459 & $3.35 \times 10^{-02} \pm 7.96 \times 10^{-02}$ & $4.50 \times 10^{-01} \pm 6.88 \times 10^{-01}$ & $\uparrow$ \\
\hline Malonate & 3.134 & $1.74 \times 10^{-02} \pm 7.29 \times 10^{-03}$ & $4.98 \times 10^{-02} \pm 2.56 \times 10^{-02}$ & $\uparrow$ \\
\hline Succinate & 2.409 & $1.91 \times 10^{-01} \pm 1.63 \times 10^{-01}$ & $9.70 \times 10^{-01} \pm 1.12 \times 10^{+00}$ & $\uparrow$ \\
\hline Pyruvate & 2.380 & $1.22 \times 10^{-01} \pm 1.48 \times 10^{-01}$ & $3.38 \times 10^{-01} \pm 3.91 \times 10^{-01}$ & $\uparrow$ \\
\hline Acetate & 1.916 & $7.24 \times 10^{-01} \pm 5.33 \times 10^{-01}$ & $4.73 \times 10^{+00} \pm 3.12 \times 10^{+00}$ & $\uparrow$ \\
\hline \multicolumn{5}{|l|}{ SCFAs } \\
\hline Propionate & 2.200 & $1.56 \times 10^{-02} \pm 9.42 \times 10^{-03}$ & $4.21 \times 10^{-01} \pm 7.61 \times 10^{-01}$ & $\uparrow$ \\
\hline Butyrate & 2.150 & $5.03 \times 10^{-02} \pm 1.95 \times 10^{-02}$ & $1.62 \times 10^{-01} \pm 2.68 \times 10^{-01}$ & $\uparrow$ \\
\hline 2-Hydroxyisovalerate & 0.837 & $2.01 \times 10^{-02} \pm 1.02 \times 10^{-02}$ & $3.87 \times 10^{-02} \pm 2.56 \times 10^{-02}$ & $\uparrow$ \\
\hline \multicolumn{5}{|l|}{ Amino acids } \\
\hline Tryptophan & 7.749 & $1.25 \times 10^{-02} \pm 5.95 \times 10^{-03}$ & $9.20 \times 10^{-03} \pm 6.66 \times 10^{-03}$ & $\downarrow$ \\
\hline Phenylalanine & 7.391 & $9.46 \times 10^{-02} \pm 3.15 \times 10^{-02}$ & $6.80 \times 10^{-02} \pm 3.43 \times 10^{-02}$ & $\downarrow$ \\
\hline Tyrosine & 7.191 & $6.77 \times 10^{-02} \pm 2.53 \times 10^{-02}$ & $4.54 \times 10^{-02} \pm 3.51 \times 10^{-02}$ & $\downarrow$ \\
\hline Glutamate & 2.354 & $3.07 \times 10^{-01} \pm 1.28 \times 10^{-01}$ & $1.77 \times 10^{-01} \pm 8.86 \times 10^{-02}$ & $\downarrow$ \\
\hline
\end{tabular}




$\begin{array}{cllll}\text { Proline } & 2.017 & 2.95 \times 10^{-02} \pm 1.19 \times 10^{-02} & 4.70 \times 10^{-02} \pm 2.88 \times 10^{-02} & \uparrow \\ \text { Isoleucine } & 1.012 & 8.27 \times 10^{-02} \pm 3.54 \times 10^{-02} & 6.91 \times 10^{-02} \pm 8.39 \times 10^{-02} & \downarrow \\ \text { Leucine } & 0.962 & 4.65 \times 10^{-01} \pm 1.95 \times 10^{-01} & 3.66 \times 10^{-01} \pm 3.43 \times 10^{-01} & \downarrow\end{array}$

\section{Nitrogenous bases}

$\begin{array}{ccccc}\text { NAD }+ & 9.341 & 2.22 \times 10^{-03} \pm 1.58 \times 10^{-03} & 1.83 \times 10^{-04} \pm 1.28 \mathrm{x}^{-03} & \downarrow \\ \text { Nicotinate } & 8.943 & 1.63 \times 10^{-03} \pm 1.30 \times 10^{-03} & 4.77 \times 10^{-03} \pm 1.92 \times 10^{-03} & \uparrow \\ \text { Uracil } & 7.543 & 1.32 \times 10^{-02} \pm 6.01 \times 10^{-03} & 3.12 \times 10^{-02} \pm 3.06 \times 10^{-02} & \uparrow \\ \text { Inosine } & 6.098 & 3.61 \times 10^{-03} \pm 7.46 \times 10^{-03} & 8.12 \times 10^{-04} \pm 1.48 \times 10^{-03} & \downarrow\end{array}$

\section{Sugars}

Glucose

$8.27 \times 10^{-02} \pm 6.48 \times 10^{-02}$

$1.67 \times 10^{-01} \pm 1.28 \times 10^{-01}$

$\uparrow$

Maltose

3.298

$1.17 \times 10^{+00} \pm 6.51 \times 10^{-01}$

$6.27 \times 10^{-01} \pm 5.10 \times 10^{-01}$

\section{Others}

$\begin{array}{ccccc}\text { Urocanate } & 6.410 & 6.15 \times 10^{-04} \pm 1.45 \times 10^{-03} & 3.84 \times 10^{-03} \pm 4.07 \times 10^{-03} & \uparrow \\ \text { Kynurenine } & 6.812 & 5.72 \times 10^{-02} \pm 2.33 \times 10^{-02} & 1.94 \times 10^{-02} \pm 1.84 \times 10^{-02}\end{array}$
sn-Glycero-3-

phosphocholine

Sarcosine

2-Aminoadipate
3.229

2.757

2.241

3-Methyl-2-
$2.45 \times 10^{-02} \pm 1.11 \times 10^{-02}$

$2.99 \times 10^{-02} \pm 1.78 \times 10^{-02}$

$8.66 \times 10^{-02} \pm 6.09 \times 10^{-02}$
$1.35 \times 10^{-02} \pm 1.72 \times 10^{-02}$

$1.17 \times 10^{-02} \pm 6.89 \times 10^{-03}$

$2.58 \times 10^{-01} \pm 1.55 \times 10^{-01}$

$\uparrow$

532 Values are expressed as means of $\mathrm{mmol} / \mathrm{l} \pm$ standard deviation.

533 TMA, Trimethylamine; SCFAs, short chain fatty acids. 
535

536

a

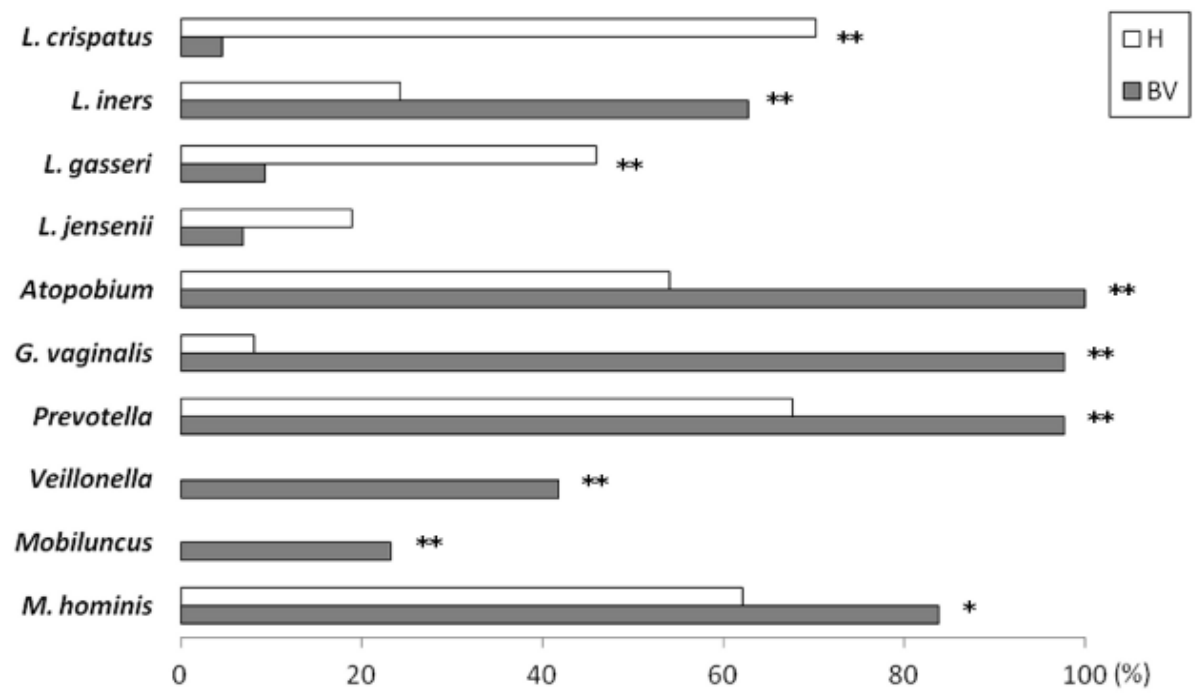

b

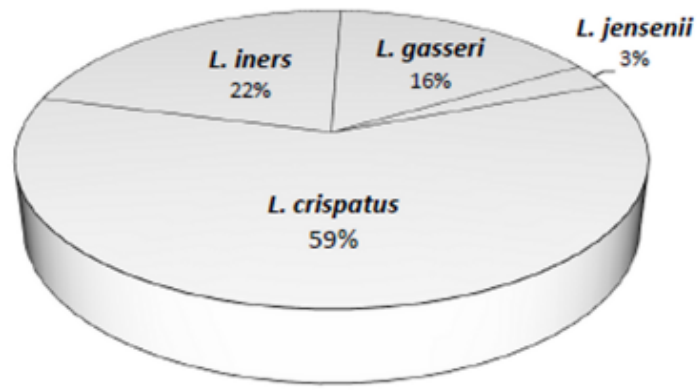

H

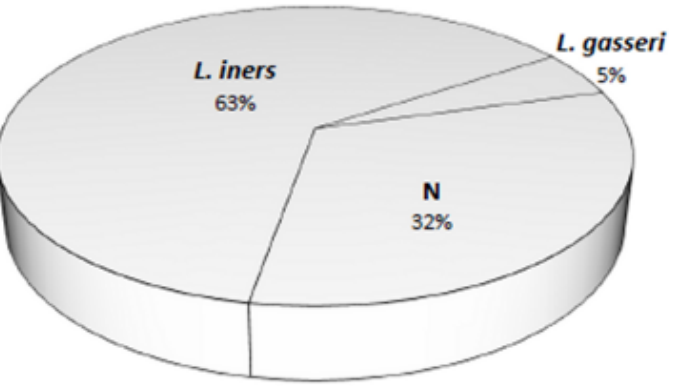

BV 
540 Figure 2

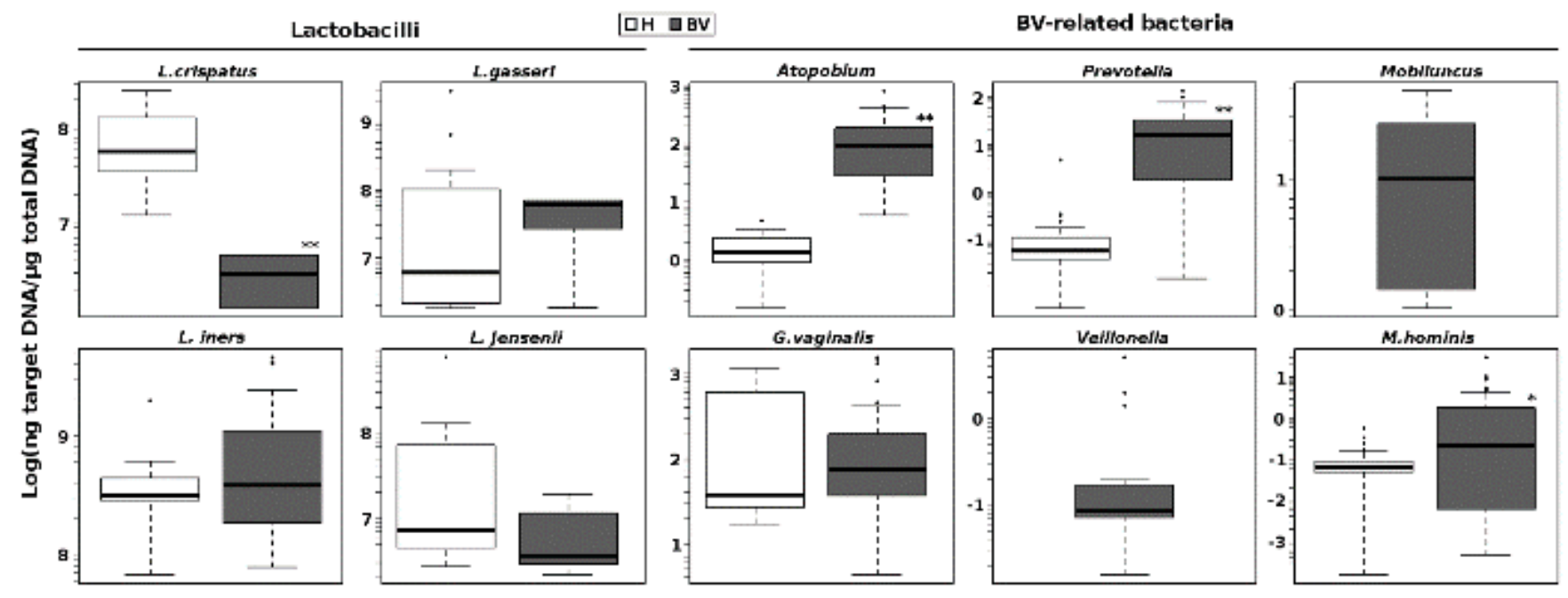

541 
$543 \quad$ Figure 3

544

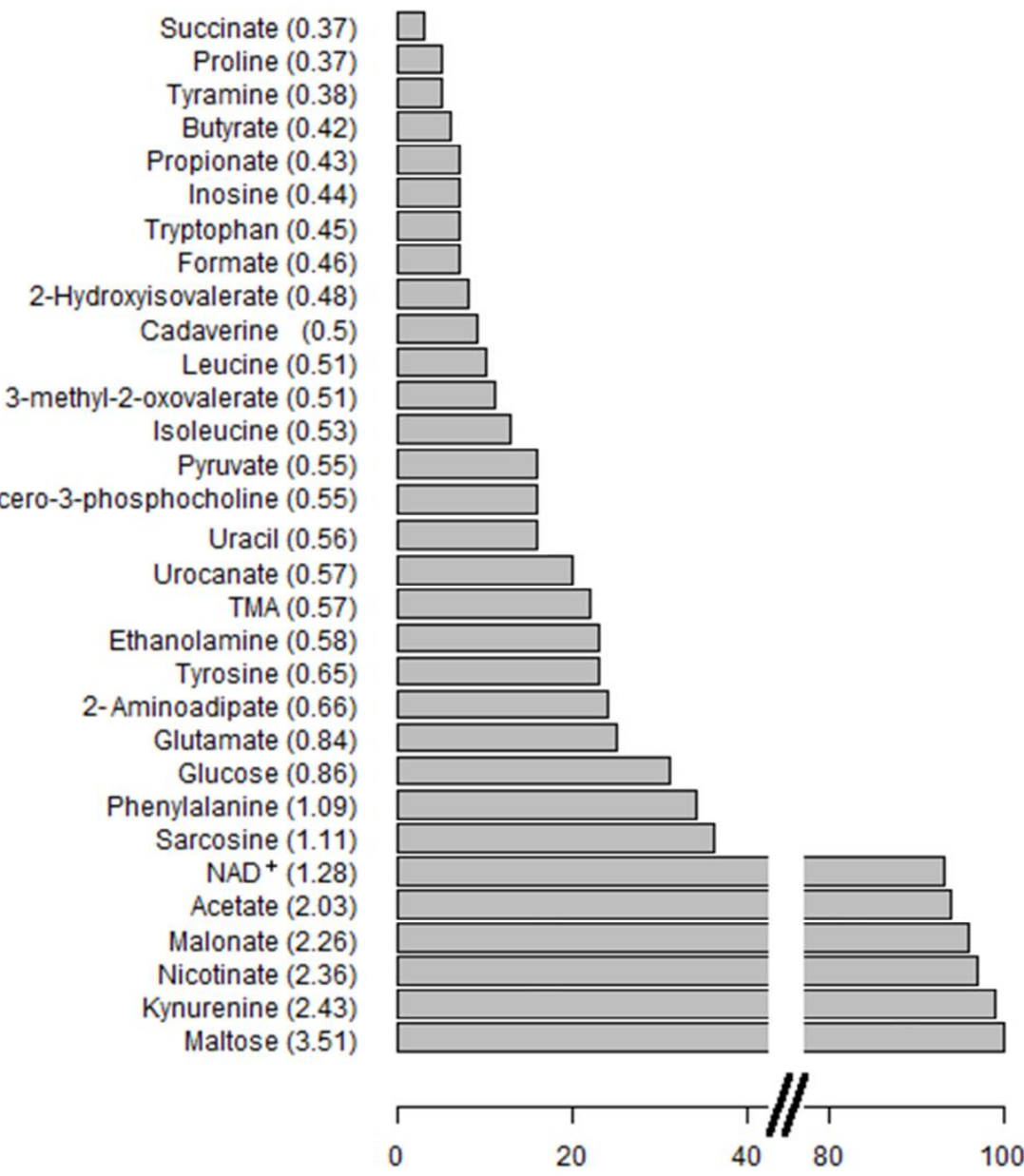

